\title{
Intravenous Immunoglobulin (IVIg) Utilization in Immune Thrombocytopenia (ITP): A Multi-Center, Retrospective Review
}

\author{
Cyrus C. Hsia $\cdot$ Yang Liu $\cdot$ Kathleen Eckert • \\ Neerav Monga $\cdot$ Julia Elia-Pacitti $\cdot$ Nancy M. Heddle
}

Published online: 22 January 2015

(C) The Author(s) 2015. This article is published with open access at Springerlink.com

\begin{abstract}
Introduction Intravenous immunoglobulin (IVIg) is an immune thrombocytopenia (ITP) therapy, which is associated with toxicities, limited availability, increasing utilization, and high cost. This study aimed to assess short- and long-term IVIg utilization in patients with ITP at two tertiary care centers in Ontario, Canada, to determine the proportion of IVIg used in ITP compared with all usage, and to forecast IVIg demand in ITP.

Methods Records from all adult ITP patients who received IVIg between January 1, 2003, and September 30, 2012, at Hamilton Health Sciences and London Health Sciences Centre were reviewed retrospectively.

Results During the study period, 383 adult ITP patients (mean age 51.3 years) received a total of 2,098 IVIg infusions (London 547 infusions in 150 patients; Hamilton 1,551 infusions in 233 patients). ITP accounted for 5.6 and $9.1 \%$ of all IVIg usage in London and Hamilton,
\end{abstract}

Electronic supplementary material The online version of this article (doi:10.1007/s40801-015-0009-6) contains supplementary material, which is available to authorized users.

C. C. Hsia · K. Eckert

London Health Sciences Centre, London, ON, Canada

C. C. Hsia $(\square)$

Division of Hematology, Department of Medicine, London Health Sciences Centre, Room E6-219, Victoria Hospital, 800 Commissioners Road East, London, ON N6A 4G5, Canada

e-mail: cyrus.hsia@1hsc.on.ca

Y. Liu - N. M. Heddle

Department of Medicine, McMaster University,

Hamilton, ON, Canada

N. Monga $\cdot$ J. Elia-Pacitti

GlaxoSmithKline Inc., Mississauga, ON, Canada respectively. The treatments included $264(53.7 \%)$ acute, $172(35.0 \%)$ short-term, and $56(11.4 \%)$ long-term treatments. The amounts of IVIg used for short- and longterm treatment of ITP are forecasted to be approximately 5,000 and $11,000 \mathrm{~g}$ per year, respectively, up to 2018 . Together, these two centers represent $19.9 \%$ of the provincial IVIg utilization. Assuming similar patient populations and practice patterns in Ontario, the overall provincial cost of IVIg use in ITP may be as high as $\$ 5$ million annually.

Conclusion Short- and long-term IVIg utilization for ITP will remain an expensive resource within the Ontario provincial health care system. Physicians and policy makers should reflect on the impact of treating ITP with IVIg and should consider alternatives, where appropriate, to improve patient quality of life and decrease economic costs.

\section{Key Points}

The amounts of intravenous immunoglobulin (IVIg) used for short- and long-term treatment of immune thrombocytopenia (ITP) are forecasted to be approximately 5,000 and 11,000 g per year, respectively, up to 2018 at these two tertiary care centers.

The estimated provincial cost of IVIg use in ITP may be as high as $\$ 5$ million annually.

Physicians and policy makers should reflect on the impact of treating ITP with IVIg and should consider alternatives, where appropriate, to improve patient quality of life and decrease economic costs. 


\section{Introduction}

Immune thrombocytopenia (ITP) is a heterogeneous autoimmune disorder characterized by the presence of platelet autoantibodies, low platelet counts, and an increased risk of bleeding $[1,2]$. Some patients will present with asymptomatic thrombocytopenia, while others will experience bleeding complications, which range in severity from skin bruises to fatal intracranial hemorrhage. ITP can be classified by duration as newly diagnosed (acute), persistent (lasting 3-12 months), or chronic (lasting $\geq 12$ months) [3].

Treatments for patients with ITP are aimed at preventing serious bleeding, improving quality of life, and achieving a safe platelet count [4]. For newly diagnosed patients, treatment with a brief course of corticosteroids, intravenous anti-D, or intravenous immunoglobulin (IVIg) results in rapid yet transient responses. Durable remissions of more than 6 months occur in fewer than $20 \%$ of patients following first-line therapy with prednisone $[5,6]$. Higher durable response rates from 59 to $75 \%$ may occur with high-dose dexamethasone, depending on the age of the patients studied and the duration of follow-up [7-9]. Chronic maintenance therapy with corticosteroids is limited by toxicities, such as neuropsychiatric symptoms, glucose intolerance, and osteoporosis; hence, other therapies are often used in relapsed or refractory ITP patients to achieve a hemostatic platelet count, thereby preventing or minimizing bleeding complications. Treatment choices include thrombopoietin receptor agonists, immunosuppressive agents, and IVIg [1, 2]. IVIg, a blood product manufactured from pooled human plasma, is a therapeutic option in the acute and chronic management of patients with ITP.

Patients with ITP are among the highest users of IVIg in Canada, representing 10-17\% of utilization for all indications $[10,11]$. IVIg administered at a dose of $1-2 \mathrm{~g} / \mathrm{kg}$ causes rapid transient increases in platelet counts in over $80 \%$ of patients [12]; however, platelet counts generally return to pretreatment levels within 4 weeks. While repeated infusions of IVIg at regular intervals may be useful as maintenance therapy for patients with ITP who require ongoing treatment because of bleeding, where a reasonable alternative exists, persistent use should be limited because of finite supplies and high associated costs [13-15]. Further, IVIg is associated with bothersome side effects, including headache, nausea, flushing, fevers, chills, fatigue, and diarrhea [1]. Less commonly, it may result in more serious complications, including anaphylaxis, hemolysis, thrombosis, renal failure, and aseptic meningitis [1, 4, 16, 17].

Over the past 5 years, in the province of Ontario, IVIg utilization for all indications has risen to 1.56 million units per year at a cost of $\$ 97.9$ million in 2010/2011, representing an increase of $44 \%$ in the number of units and a $53 \%$ increase in costs [15]. Given the growth in the utilization of IVIg in various clinical conditions, it is forecasted that the supply may not be able to meet the demand [18]. In 2006, the Ontario Ministry of Health and Long Term Care (MOHLTC) identified the unsustainable increases in IVIg utilization as a key priority [18]; however, there is currently no provincial mechanism for routinely tracking and accurately quantifying and characterizing IVIg use.

This study aimed to assess the utilization of IVIg in patients with ITP at two large tertiary care centers; determine the extent of short- and long-term utilization; assess the proportion of IVIg usage for ITP compared with all indications; compare utilization between these two unique centers; and forecast future demand.

\section{Methods}

\section{Study Design}

A retrospective analysis of all adult patients who received IVIg for the treatment of ITP at these two participating centers during the study period was performed. Local institutional ethics review boards at both sites approved this study.

\section{Study Population}

Patients were eligible for inclusion if they met the following criteria: age 18 years or older; a diagnosis of ITP; and receipt of at least one dose of IVIg for the treatment of ITP between January 1, 2003, and September 30, 2012, at either of two large tertiary care centers, Hamilton Health Sciences (HHS) and London Health Sciences Centre (LHSC). Patients were excluded if an alternate cause of thrombocytopenia other than ITP was identified.

\section{Data Collection}

Data collected at both centers included the date of birth, sex, weight (if available), date of IVIg infusion, and amount of IVIg administered.

At HHS, potential ITP patients were identified and cases were confirmed by chart review. The Transfusion Registry for Utilization, Surveillance and Tracking (TRUST) database, developed by the McMaster Transfusion Research Program (MTRP), was used as the primary source of data extraction in Hamilton. TRUST comprises data primarily from two sources of electronic data capture: Meditech 
(Meditech Circle, Westwood, MA, USA), and the Discharge Abstract Database (DAD). Meditech is a laboratory information system used at HHS, which houses laboratory values and transfusion/infusion product information. The DAD is the electronic database at both institutions that collects clinical data for the Canadian Institute for Health Information (CIHI). It was used to identify patients with a diagnosis of ITP, extract patient information, and determine the indication for IVIg, etc. To confirm the diagnosis, information was obtained from patients' clinic charts and electronic medical records.

Patient diagnoses are coded using the CIHI International Classification of Diseases and Related Health Problems, 10th Revision, Canada (ICD-10-CA). During the study period, two of the three Hamilton hospitals issued IVIg from the Transfusion Medicine Laboratory; hence, the information on IVIg disposition was in the laboratory information system (Meditech) and had been captured in TRUST. The third hospital (at the McMaster site) issued IVIg from the Transfusion Medicine Laboratory between 2009 and 2012; however, from 2002 to 2009, the IVIg product was issued from the Pharmacy and recorded manually. The information on these paper logs was entered into a spreadsheet and cross-linked with the DAD data to identify all potential ITP patients. A chart review was then performed on all potential ITP patients to confirm their diagnosis and eligibility for final inclusion in the analysis.

At LHSC, the Transfusion Medicine Laboratory information system contained all infusion episodes and data on IVIg utilized within the study period, including all inpatient and outpatient IVIg utilization at all hospital sites (i.e., University Hospital and Victoria Hospital of LHSC, and St. Joseph's Health Care Centre). This database did not identify ICD-10 codes, but all IVIg requested through the Transfusion Medicine Laboratory required an IVIg request form, which documented the indications. All ITP-related IVIg requests were therefore identified from these request forms. Further, one of the investigators $(\mathrm{CH})$ retrospectively reviewed the clinical data from the recipients' patient electronic records to differentiate and confirm the diagnosis of ITP versus an error in coding due to another cause of thrombocytopenia. Where electronic records were not available, paper charts were reviewed. Once confirmed, all eligible patient data were captured and reviewed. The de-identified data were submitted to the MTRP, where the data from the two sites were combined and analyzed.

The primary outcome of this study was a description of the proportion of patients using IVIg in the short-term and long-term chronic ITP settings, and the corresponding durations of therapy. Secondary outcomes included the number of IVIg infusions administered; number of IVIg infusions administered per patient; average total amount administered per infusion, per course, and per treatment period; average number of infusions and courses given per treatment period; days between courses; percentage of total IVIg use (for all indications) that was used for ITP; estimated IVIg product usage cost per year; and future forecasts of IVIg utilization from 2012 to 2018.

\section{Definitions}

To determine the primary outcome of quantifying shortand long-term utilization, definitions of acute, short-term and long-term treatments were developed on the basis of clinical judgment by experts in the treatment of ITP, both individually and by consensus as part of an advisory board meeting sponsored by GlaxoSmithKline Inc. Infusions of IVIg were grouped to form courses, and courses were grouped to form treatment periods, which were then classified as acute, short-term, or long-term (Fig. 1). A "course" was defined as the number of IVIg infusions administered within a 5-day period. This definition allowed for variable practice patterns, as physicians often order IVIg 1-2 g/kg divided over 1,2 , or 5 days. A "treatment period" was defined as a collection of courses where consecutive courses were given within a 6-month (182day) time frame of each other. Thus, a single treatment period could last for years if consecutive courses during that treatment period were given within 6 months of each other. Consecutive courses that were given more than 6 months apart were considered to be in separate treatment periods (i.e., a patient may have been treated in multiple treatment periods). An "acute treatment" was defined as involving only one course in the treatment period. Clinically, for example, this could correspond to using IVIg once for emergency treatment of bleeding associated with low platelet counts. A "short-term treatment" contained $2-5$ courses, possibly representing a longer bridging treatment until another therapy started to work, and a "long-term treatment" was defined as containing six or more courses in that treatment period, perhaps denoting maintenance therapy.

\section{Statistical Analysis}

LHSC data were sent to HHS for centralized analysis. A data dictionary was developed for the data from each site, and variable names were mapped to standardize data coding. SAS 9.3 software (SAS Institute, Cary, NC, USA) was used to perform this cross-mapping and for data analysis. Descriptive statistics were used to determine the proportion of IVIg being used for patients with ITP compared with all other indications. IVIg utilization data were analyzed by course and treatment period, as defined above. Time series forecasting utilization for 2012-2018 was performed using a stepwise autoregressive method (SAS 9.3, PROC 
Fig. 1 Summary of steps used to classify intravenous immunoglobulin (IVIg) infusions into courses, treatment periods, and acute, short-term, and long-term treatment periods

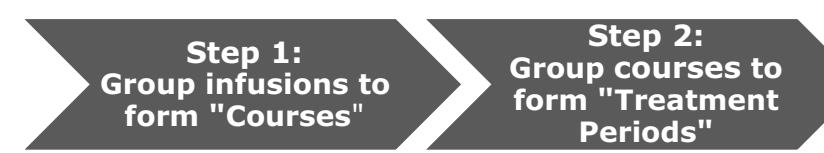

- Course $=1$ to 5 IVIg infusions which occur within 5 days
-Treatment Period= a collection of consecutive courses that are separated by less than 6 months (or $\leq 182$ days)

-i.e. consecutive courses that are $>6$ months apart are considered in 2 separate treatments periods

Step 3:
Classify
"Treatment
Periods"
treatment period
FORECAST) with the history data from 2003 to 2011. The stepwise autoregressive method (STEPAR) that was used combines a time trend regression with an autoregressive model for departures from the trend.

\section{Results}

There were 383 adult ITP patients who received a total of 2,098 IVIg infusions at the two major Ontario tertiary care centers participating in this retrospective study between January 1, 2003, and September 30, 2012 (Tables 1, 2). Comparing the two centers, in London, 547 infusions were given to 150 patients, and in Hamilton, 1,551 infusions were given to 233 patients (Table 2). Despite this difference, the proportions of male and female recipients were similar at the two sites. The mean age of the patients at the first IVIg infusion was 51.3 years, with a range of ages from 18 to 96 years of age (Table 1). The mean weight $(80.4 \mathrm{~kg})$ was available and calculated from 195 recipients (London 81/150, 54 \%; Hamilton 114/233, $49 \%$ ) and was also similar at the two centers (Table 1). Patients received, on average, 5.5 IVIg infusions (London 3.6, Hamilton 6.7; range $1-196$ ) at $0.96 \mathrm{~g} / \mathrm{kg}$ per infusion (Table 2).

When IVIg infusions were combined into courses, there were a total of 1,603 courses of therapy given (London 428 courses; Hamilton 1,175 courses), with a mean of 4.2 courses given per patient and 1.3 infusions given per course (Table 2). The majority of patients received one or two infusions per course, which was similar at the two centers. Further, the majority of patients receiving infusions at both centers received approximately 1 or $2 \mathrm{~g} / \mathrm{kg}$ per course, with $1 \mathrm{~g} / \mathrm{kg}$ being the most commonly prescribed amount, followed by $2 \mathrm{~g} / \mathrm{kg}$.

When grouped into treatment periods (as defined earlier), there were a total of 492 treatment periods (London 172, Hamilton 320) with $264(53.7 \%)$ defined as acute, $172(35.0 \%)$ short-term, and 56 (11.4\%) long-term treatments (Tables 3, 4). The majority of patients, 306 $(79.9 \%)$, received all of their IVIg infusions in one treatment period, but some patients had up to six treatment periods. The average number of courses given in a treatment period was 3.3 (London 2.5, Hamilton 3.7; range 1-99). This correlated with an average 4.3 IVIg infusions given per treatment period (London 3.2, Hamilton 4.8; range 1-196) [Table 3]. The number of days between consecutive courses within these treatment periods was 29.5 days (London 32.2, Hamilton 28.7), and the number of days between consecutive treatment periods, for those patients who received IVIg in more than one treatment period, was 636 days and was similar at the two centers (London 632, Hamilton 637).

The number of grams of IVIg used in London and Hamilton for all indications at both sites was seen to be

Table 1 Demographic characteristics by site and overall

\begin{tabular}{llll}
\hline Characteristic & London, $n=150$ & Hamilton, $n=233$ & Total, $n=383$ \\
\hline Male sex $[n(\%)]$ & $56(37.3)$ & $88(37.8)$ & $144(37.6)$ \\
Age at the first IVIg infusion [years; mean/SD] & $54.0 / 20.04$ & $49.5 / 19.16$ & $51.3 / 19.60$ \\
Patients with available weight data $[n]$ & 81 & 114 & 195 \\
Average weight at time of first infusion $[\mathrm{kg} ;$ mean/SD] & $80.1 / 18.23$ & $80.6 / 20.10$ & $80.4 / 19.30$ \\
\hline
\end{tabular}

IVIg intravenous immunoglobulin, $S D$ standard deviation

a Age was calculated using the birth year of each patient, so for some patients the age may have been overcalculated by 1 year 
Table 2 Intravenous immunoglobulin (IVIg) usage: infusions and courses by site and overall

\begin{tabular}{llll}
\hline & London, $n=150$ & Hamilton, $n=233$ & Total, $n=383$ \\
\hline${\text { Total number of IVIg infusions }{ }^{\mathrm{a}}}_{\text {Number of IVIg infusions per patient [mean/SD] }}$ & 547 & 1,551 & 2,098 \\
Amount per infusion [g/kg; mean/SD] & $3.6 / 6.79$ & $6.7 / 15.65$ & $5.5 / 13.00$ \\
Total number of IVIg courses & $0.91 / 0.1902$ & $0.98 / 0.2181$ & $0.96 / 0.2121$ \\
Number of IVIg courses per patient [mean/SD] & 428 & 1,175 & 1,603 \\
Number of infusions per course [mean/SD] & $2.9 / 6.39$ & $5.0 / 9.62$ & $4.2 / 8.56$ \\
Total grams of IVIg & $1.3 / 0.50$ & $1.3 / 0.49$ & $1.3 / 0.49$ \\
\hline
\end{tabular}

$S D$ standard deviation

${ }^{a}$ Infusions occurring on the same day were counted as one infusion

Table 3 Intravenous immunoglobulin (IVIg) usage categorized by treatment type by site and overall

\begin{tabular}{llll}
\hline & London, $n=150$ & Hamilton, $n=233$ & Total, $n=383$ \\
\hline Total number of IVIg treatment periods $(\%)$ & 172 & 320 & 492 \\
$\quad$ Acute treatment $(\%)$ & $101(58.7)$ & $163(50.9)$ & $264(53.7)$ \\
Short-term treatment $(\%)$ & $60(34.9)$ & $112(35.0)$ & $172(35.0)$ \\
Long-term treatment $(\%)$ & $11(6.4)$ & $45(14.1)$ & $56(11.4)$ \\
Number of IVIg treatment periods received per patient [mean/SD] & $1.1 / 0.44$ & $1.4 / 0.78$ & $1.3 / 0.68$ \\
Courses per treatment period [mean/SD] & $2.5 / 5.86$ & $3.7 / 7.79$ & $3.3 / 7.19$ \\
Infusions per treatment period [mean/SD] & $3.2 / 6.13$ & $4.8 / 12.85$ & $4.3 / 11.00$ \\
Total grams per treatment period [mean/SD] & $226.6 / 475.08$ & $378.4 / 1,121.75$ & $325.4 / 949.41$ \\
\hline
\end{tabular}

$S D$ standard deviation

Table 4 Intravenous immunoglobulin (IVIg) utilization analysis by treatment type (overall population)

\begin{tabular}{llll}
\hline Key findings & $\begin{array}{l}\text { Long-term treatment: } \\
6 \text { or more courses }\end{array}$ & $\begin{array}{l}\text { Short-term treatment: } \\
2-5 \text { courses }\end{array}$ & $\begin{array}{l}\text { Acute treatment: } \\
1 \text { course }\end{array}$ \\
\hline Number of classified treatment periods $(\%)$ & $56(11.4)$ & $172(35.0)$ & $264(53.7)$ \\
Patients $[n]$ & 52 & 152 & 228 \\
Total IVIg amount per treatment type [g; mean/SD] & $1,559.5 / 2,482.91$ & $281.6 / 136.20$ & $92.1 / 36.11$ \\
Average number of infusions per treatment period [mean/SD] & $19.9 / 27.90$ & $3.8 / 1.66$ & $1.3 / 0.50$ \\
Days of therapy per treatment period [mean/SD] & $427 / 385.18$ & $84.2 / 79.44$ & \\
\hline
\end{tabular}

$S D$ standard deviation

trending upward from $144,605 \mathrm{~g}$ in 2003 to $245,763 \mathrm{~g}$ in 2012 (see Table A1 in the Electronic Supplementary Material). Utilization for ITP also increased, but, relative to all other indications, the proportion of IVIg used for adult ITP patients remained relatively stable (see Table A1). The actual and forecasted IVIg usage in grams is provided in Table 5. The proportion of IVIg given to adult ITP patients ranged from 5.6 to $9.1 \%$ and was generally lower in London than in Hamilton (see Table A2 in the Electronic Supplementary Material). In total, $160,076 \mathrm{~g}$ of IVIg was administered in 2,098 infusions for patients with ITP, representing $7.9 \%$ of the overall amount of IVIg usage for all indications, which differed between the centers (London $4.6 \%$, Hamilton $10.3 \%$ ) [see Table A1].

\section{Discussion}

This 10-year, retrospective study at two tertiary care centers analyzed data from both sites to provide comprehensive information on IVIg utilization in adult ITP patients. These two large centers represent $19.9 \%$ of the total IVIg utilization (for all indications) in the province of Ontario. During the study period, there were 383 adult ITP patients who received a total of $160,076 \mathrm{~g}$ of IVIg in 2,098 infusions. This represented $7.9 \%$ of the total IVIg utilization for all indications-somewhat lower than the 10-17\% previously reported in Atlantic Canada [10, 11]. On average, there were roughly 1.5 times more women than men who received IVIg, which is in keeping with the higher 
Table 5 Annual actual and forecasted intravenous immunoglobulin (IVIg) short- and long-term usage in patients with immune thrombocytopenia

\begin{tabular}{|c|c|c|c|c|c|c|}
\hline \multirow[t]{3}{*}{ Year } & \multicolumn{6}{|c|}{ Amount of IVIg used [g] } \\
\hline & \multicolumn{2}{|l|}{ London } & \multicolumn{2}{|l|}{ Hamilton } & \multicolumn{2}{|l|}{ Total } \\
\hline & $\begin{array}{l}\text { Short-term } \\
\text { treatment }\end{array}$ & $\begin{array}{l}\text { Long-term } \\
\text { treatment }\end{array}$ & $\begin{array}{l}\text { Short-term } \\
\text { treatment }\end{array}$ & $\begin{array}{l}\text { Long-term } \\
\text { treatment }\end{array}$ & $\begin{array}{l}\text { Short-term } \\
\text { treatment }\end{array}$ & $\begin{array}{l}\text { Long-term } \\
\text { treatment }\end{array}$ \\
\hline 2003 & 1,460 & 600 & 2,950 & 6,090 & 4,410 & 6,690 \\
\hline 2004 & 140 & 1,260 & 3,467 & 11,353 & 3,607 & 12,613 \\
\hline 2005 & 1,180 & 0 & 4,422 & 7,838 & 5,602 & 7,838 \\
\hline 2006 & 2,397 & 1,260 & 3,248 & 5,695 & 5,645 & 6,955 \\
\hline 2007 & 1,300 & 1,020 & 3,503 & 11,137 & 4,803 & 12,157 \\
\hline 2008 & 1,295 & 960 & 2,165 & 6,586 & 3,460 & 7,546 \\
\hline 2009 & 1,977 & 1,600 & 1,810 & 6,500 & 3,787 & 8,100 \\
\hline 2010 & 2,395 & 2,785 & 4,580 & 2,390 & 6,975 & 5,175 \\
\hline 2011 & 1,805 & 2,980 & 2,190 & 12,515 & 3,995 & 15,495 \\
\hline $2012^{\mathrm{a}}$ & 2,270 & 2,801 & 2,648 & 7,542 & 4,918 & 10,343 \\
\hline $2013^{\mathrm{a}}$ & 2,414 & 3,085 & 2,548 & 7,492 & 4,962 & 10,577 \\
\hline $2014^{\mathrm{a}}$ & 2,558 & 3,368 & 2,447 & 7,442 & 5,005 & 10,810 \\
\hline $2015^{a}$ & 2,702 & 3,651 & 2,347 & 7,393 & 5,049 & 11,044 \\
\hline $2016^{\mathrm{a}}$ & 2,846 & 3,934 & 2,247 & 7,343 & 5,093 & 11,277 \\
\hline $2017^{a}$ & 2,990 & 4,218 & 2,147 & 7,294 & 5,137 & 11,512 \\
\hline $2018^{a}$ & 3,134 & 4,501 & 2,047 & 7,244 & 5,181 & 11,745 \\
\hline
\end{tabular}

${ }^{a}$ The 2012-2018 data were forecasted using the complete annual data from 2003 to 2011

prevalence of ITP in women than in men [19], with estimated female to male ratios of $1.2: 1$ and $1.7: 1$ being previously reported $[20,21]$. The range of patient ages (18-96 years) was broad, reflecting the diverse patient population who develop ITP and require treatment [22].

IVIg utilization patterns were generally similar at the two study centers, including the age of the patients treated, the proportions of male and female recipients, and the amount of IVIg infused. However, there were more IVIg infusions given to more ITP patients in Hamilton than in London. These two sites are independent of one another and have dissimilar catchment areas and referral practices. Hamilton has a very specialized dedicated ITP practice, with referrals from other tertiary care centers to its ITP clinics, reflecting a potentially sicker, usually more heavily pretreated population, who may require longer and perhaps more intensive treatments. London has a more communitybased referral pattern, where patients are typically seen by a specialist for the first time after diagnosis by a family physician or emergency room physician. Despite the clinical heterogeneity in the two patient populations, our results suggest similar practice patterns across these two centers.

The majority of patients received approximately 1 or $2 \mathrm{~g} / \mathrm{kg}$ per course, with $1 \mathrm{~g} / \mathrm{kg}$ being the most commonly prescribed dose at both centers, followed by $2 \mathrm{~g} / \mathrm{kg}$. This is in keeping with infusions used in clinical practice and guidelines $[1,2]$. The recently updated American Society of Hematology evidence-based guidelines for ITP recommend $1 \mathrm{~g} / \mathrm{kg}$ as an initial dose [2]. However, it is noted that patients who fail to respond to $1 \mathrm{~g} / \mathrm{kg}$ may respond to a higher dose of $2 \mathrm{~g} / \mathrm{kg}$ [12]. At both centers, the majority of IVIg was given for acute treatment of ITP (as per our definition). Less IVIg was used for short-term treatment, and it was infrequently used for long-term treatment. It is possible that acute treatments were given for ITP patients who required therapy for active bleeding, a bridge to a procedure or surgery with an increased bleeding risk (i.e., splenectomy), or a trial to assess platelet response. Compared with the London site, Hamilton treated a larger cohort of ITP patients with slightly more IVIg infusions per patient, gave more courses per patient, had a higher proportion of ITP patients relative to those with other indications, and administered more grams of IVIg for ITP relative to other indications, as would be expected with its highly specialized ITP referral pattern.

Unlike acute treatments, short-term and long-term therapy with IVIg may be provided to patients with the intent of using it as the sole maintenance therapy or as a longer bridging solution until another therapy works to control ITP. From the present data, we can predict the impact of IVIg utilization in adult ITP patients requiring short- and long-term treatments (Table 5). The total amount of IVIg used for long-term treatment of ITP at these two centers 
from 2012 to 2018 is forecasted to remain at approximately $11,000 \mathrm{~g}$ per year, at an estimated cost of $\$ 693,000$ per year, based on a unit price of approximately $\$ 63$ per gram in 2011, published in Bloody Easy 3 by Callum et al. [23]. However, short-term IVIg treatments make up an additional $172(35 \%)$ of the total number of IVIg treatment periods. With $281.6 \mathrm{~g}$ administered per short-term treatment period, this could contribute to an estimated additional 5,000 $\mathrm{g}$ of IVIg being used, costing $\$ 315,000$ per year, for a total of roughly $\$ 1$ million per year for both short- and long-term treated patients. The estimated costs from these two centers represent $19.9 \%$ of the overall provincial utilization. Assuming that the patient populations and practice patterns are similar across Ontario, the overall cost in the province for short- and long-term treatments of ITP may be upward of fivefold greater, or $\$ 5$ million per year. It was not feasible to include any kind of in-depth resource utilization discussion, since various components, such as nursing costs, chair time, and monitoring for reactions, were not included as part of this study. Thus, a complete and accurate cost analysis of all components was not performed, and the true cost impact of IVIg to the health care system, based only on the product cost of IVIg, is underestimated. As forecasted by our data, IVIg usage for ITP will remain a substantial burden on the public health care budget and will continue to increase by approximately $2 \%$ per year from 2013 to 2018 .

IVIg usage for ITP should be reduced for several reasons other than its high cost. While IVIg safety and toxicity were not studied in this review, IVIg is associated with numerous potential side effects. Bothersome side effects include headache, nausea, flushing, fevers, chills, fatigue, and diarrhea (Provan et al. [1]). Less commonly, it may result in serious complications, including anaphylaxis, hemolysis, thrombosis, renal failure, and aseptic meningitis $[1,4,16,17]$. Further, IVIg is a limited resource, which is widely utilized and can impact the quality of life of patients, including the need for travel to an infusion clinic, the need to sit for several hours during the infusion, and anxiety over possible reactions. Limiting the use of IVIg for ITP is one of the items highlighted by Choosing Wisely Canada and the Canadian Hematology Society [24]. In the last 5 years, newer therapies, such as thrombopoietic mimetics, have become available to patients with ITP, and the role of these agents as an alternative to IVIg, particularly for short- and long-term treatments, requires consideration.

Study limitations include the retrospective data collection from various databases at these two centers. However, most of the data came from the laboratory information system at each site, which is considered the gold-standard data repository for blood product utilization. While these two large centers represent $19.9 \%$ (London $6.5 \%$, Hamilton $13.4 \%$ ) of total IVIg utilization (for all indications) in the province of Ontario, it is not clear if the findings are representative of the province as a whole (i.e., community/ rural settings). The thrombopoietin mimetics were approved for ITP treatments in the past few years and may have impacted the utilization of IVIg as reflected in this retrospective analysis. Further, clinical trials with these new medications at the Hamilton site, and a provincial IVIg audit [15], may have reduced the enthusiasm for using IVIg during the past few years and may have affected our estimates.

\section{Conclusion}

This retrospective review of comprehensive data at the London and Hamilton sites has helped to characterize IVIg utilization in adult ITP patients and may improve the understanding of its impact on provincial utilization and inform future clinical practice and policy decisions. With the current practice pattern, short- and long-term IVIg utilization for ITP will remain very expensive for the health care system. Physicians treating ITP and policy makers should consider the impact of treating ITP with IVIg and should consider alternatives, where appropriate, to improve both patient quality of life and economic impact.

Competing interests Cyrus C. Hsia: has been a consultant on advisory boards and received speaker honoraria from Amgen and GlaxoSmithKline Inc. Yang Liu: the McMaster Transfusion Research Program receives infrastructure funding from Canadian Blood Services and Health Canada. Kathleen Eckert: no disclosures. Neerav Monga and Julia Elia-Pacitti: employees of GlaxoSmithKline Inc. Nancy Heddle: is a consultant for Macopharma and Terumo BCT, and the McMaster Transfusion Research Program receives infrastructure funding from Canadian Blood Services and Health Canada.

Author contributions Cyrus C. Hsia, Neerav Monga, Julia EliaPacitti, and Nancy Heddle conceived the study. All authors were involved in the design of the study. Cyrus C. Hsia, Yang Liu, Kathleen Eckert, and Nancy Heddle were involved in the collection of data. Yang Liu and Neerav Monga conducted the analyses. Cyrus C. Hsia drafted the manuscript, and all authors provided comments on the drafts and have read and approved the final version submitted for publication. Cyrus C. Hsia is the guarantor for this work.

Funding This Project was funded by GlaxoSmithKline Inc.

Open Access This article is distributed under the terms of the Creative Commons Attribution Noncommercial License which permits any noncommercial use, distribution, and reproduction in any medium, provided the original author(s) and the source are credited.

\section{References}

1. Provan D, Stasi R, Newland AC, et al. International consensus report on the investigation and management of primary immune thrombocytopenia. Blood. 2010;115:168-86. 
2. Neunert C, Lim W, Crowther M, Cohen A, Solberg L Jr, Crowther MA. The American Society of Hematology 2011 evidence-based practice guideline for immune thrombocytopenia. Blood. 2011;117:4190-207.

3. Rodeghiero F, Michel M, Gernsheimer T, et al. Standardization of terminology, definitions and outcome criteria in immune thrombocytopenic purpura of adults and children: report from an international working group. Blood. 2009;113:2386-93.

4. Arnold DM, Kelton JG. Current options for the treatment of idiopathic thrombocytopenic purpura. Semin Hematol. 2007;44: S12-23.

5. Stasi R, Stipa E, Masi M, et al. Long-term observation of 209 adults with chronic idiopathic thrombocytopenic purpura. Am J Med. 1995;98(5):436-42.

6. Pamuk GE, Pamuk ON, Baslar Z, et al. Overview of 321 patients with idiopathic thrombocytopenic purpura: retrospective analysis of the clinical features and response to therapy. Ann Hematol. 2002;81(8):436-40.

7. Mashhadi MA, Kaykhaei MA, Sepehri Z, Miri-Moghaddam E. Single course of high dose dexamethasone is more effective than conventional prednisolone therapy in the treatment of primary newly diagnosed immune thrombocytopenia. DARU J Pharm Sci. 2012;20(1):7.

8. Mazzucconi MG, Fazi P, Bernasconi S, et al. Gruppo Italiano Malattie E Matologiche dell'Adulto (GIMEMA) Thrombocytopenia Working Party. Therapy with high-dose dexamethasone (HD-DXM) in previously untreated patients affected by idiopathic thrombocytopenic purpura: a GIMEMA experience. Blood. 2007;109(4):1401-7.

9. Borst F, Keuning JJ, van Hulsteijn H, Sinnige H, Vreugdenhil G. High-dose dexamethasone as a first- and second-line treatment of idiopathic thrombocytopenic purpura in adults. Ann Hematol. 2004;83(12):764-8.

10. Constantine MM, Thomas W, Whitman L, et al. Intravenous immunoglobulin utilization in the Canadian Atlantic provinces: a report of the Atlantic Collaborative Intravenous Immune Globulin Utilization Working Group. Transfusion. 2007;47:2072-80.

11. Quraishi TA, et al. IVIg and SCIg Utilization in the Atlantic Provinces: FY 2009/2010. Nova Scotia Provincial Blood Coordinating Program. http://novascotia.ca/dhw/nspbcp/docs/IVIGSCIG-Utilization-Atlantic-Provinces-FY-2009-10.pdf. Accessed 1 Jun 2014.

12. Godeau B, Caulier MT, Decuypere L, et al. Intravenous immunoglobulin for adults with autoimmune thrombocytopenic purpura: results of a randomized trial comparing 0.5 and $1 \mathrm{~g} /$ kg b.w. Br J Haematol. 1999;107(4):716-9.

13. Godeau B, Lesage S, Divine M, Wirquin V, Farcet JP, Bierling P. Treatment of adult chronic autoimmune thrombocytopenic purpura with repeated high-dose intravenous immunoglobulin. Blood. 1993;82(5):1415-21.

14. Bussel JB, Pham LC, Aledort L, Nachman R. Maintenance treatment of adults with chronic refractory immune thrombocytopenic purpura using repeated intravenous infusions of gammaglobulin. Blood. 1988;72(1):121-7.

15. ORBCoN (Ontario Regional Blood Coordinating Network) Intravenous immune globulin (IVIg) utilization audit. March 2009. http://transfusionontario.org/en/cmdownloads/categories/ reports/. Accessed 1 Jun 2014.

16. Caress JB, Kennedy BL, Eickman KD. Safety of intravenous immunoglobulin treatment. Expert Opin Drug Saf. 2010;9(6): 971-9.

17. Mitterer M, Pescosta N, Vogetseder W, Mair M, Coser P. Two episodes of aseptic meningitis during intravenous immunoglobulin therapy of idiopathic thrombocytopenic purpura. Ann Hematol. 1993;67(3):151-2.

18. Ontario IVIg Utilization Management Strategy, March 31, 2012. http:// transfusionontario.org/en/cmdownloads/categories/ivig. Accessed 1 Jun 2014.

19. Cines DB, Blanchette VS. Immune thrombocytopenic purpura. N Engl J Med. 2002;346(13):995-1008.

20. Frederiksen H, Schmidt K. The incidence of idiopathic thrombocytopenic purpura in adults increases with age. Blood. 1999;94:909-13.

21. Neylon AJ, Saunders PWG, Howard MR, Proctor SJ, Taylor PRA, The Northern Regional Haematology Group. Clinically significant newly presenting autoimmune thrombocytopenic purpura in adults: a prospective study of a population-based cohort of 245 patients. Br J Haematol. 2003;122:966-74.

22. Cines DB, Bussel JB. How I treat idiopathic thrombocytopenic purpura (ITP). Blood. 2005;106(7):2244-51.

23. Callum JL, Lin Y, Pinkerton PH, editors. Bloody easy 3: blood transfusions, blood alternatives and transfusion reactions: a guide to transfusion medicine. Toronto: Ontario Regional Blood Coordinating Network; 2011.

24. Choosing wisely Canada: Canadian Hematology Society. http:// www.choosingwiselycanada.org/wp-content/uploads/2014/09/CHSEN-web.pdf. Accessed 16 Nov 2014. 